\title{
Diamagnetism of Electron Gas on Surface of Semiconductor Nanotube
}

\author{
G.I. RASHBA
}

Department of Theoretical Physics named after I.M. Lifshits

Kharkiv National University named after V.N. Karazin, 4 Svobody Sq., Kharkiv, 61022, Ukraine

Received: 25.08.2020 \& Accepted: 27.10.2020

Doi: 10.12693/APhysPolA.139.66 *e-mail: georgiy.i.rashba@gmail.com

\begin{abstract}
The density of states of electrons on the surface of a semiconductor cylindrical nanotube and the grand thermodynamic potential in a longitudinal magnetic field are obtained in terms of the Jacobi elliptic theta function. At low temperatures, the diamagnetic moment of the electron gas on the tube in a weak magnetic field is calculated.
\end{abstract}

topics: nanotube, grand thermodynamic potential, electron gas, diamagnetic moment

\section{Introduction}

Nanotubes exhibit a whole range of important magnetic properties which, along with geometric parameters, give grounds for their use as a promising structural material for modern electronics due to the fact that miniaturization of electronic devices directly entails an increase in current densities $[1-4]$. As a result, traditional materials can no longer be used.

Twenty years before the discovery of carbon nanotubes, the quantization of the magnetic flux in a cylindrical normal tube was discovered [5]. The magnetic moment of the electron gas on its surface was then calculated. The discovery of carbon and semiconductor nanotubes was discussed in, e.g., [6] and their magnetic properties were considered in, e.g., $[1,2,7]$.

When an electron rotates around the walls of a nanotube, a magnetic response occurs, known as the orbital magnetic moment [4]. The orbital magnetism of carbon nanotubes was studied by Ajiki and Ando [8] while the magnetic response of a semiconductor nanotube was examined in [9]. The problem of the magnetization of nanostructures with cylindrical symmetry is closely related to the problem of persistent currents in quantum rings and nanotubes [10]. These currents create an orbital magnetic moment which is called diamagnetic [3].

The Aharonov-Bohm effect in nanotubes was investigated, e.g., in [11]. The exchange energy of an electron gas on a tube in a longitudinal magnetic field, in turn, was considered in [12]. The thermodynamic functions of an electron gas on the surface of a semiconductor nanotube in a magnetic field, in particular the spin magnetization of an electron gas, were investigated in [13]. Further, excitons in cylinder conductors were studied in [14] while plasmons in nanotubes with a superlattice were examined in [15]. In [16-19], the theory of the LandauSilin electron spin waves on a tube in a magnetic field was presented and it was reviewed in [20, 21]. In turn, the spectra of plasma waves in the electron gas on the surface of a semiconductor nanotube with a superlattice in a parallel magnetic field were studied in [22]. The magnetic properties in the metal conduction mode were discussed in connection with the problems of persistent currents and magnetoresistance in [23-25]. In [23], the persistent current was calculated taking into account the scattering of electrons by the $\delta$-potential of the impurity atom. Moreover, the properties of carbon nanotubes in the Luttinger model were studied in [24] while the magnetoresistance of nanotubes in a transverse magnetic field was considered in [25].

In the mentioned papers, the Landau diamagnetism was not considered within the framework of the simplest model. The model is assumed to be an infinitely long and thin tube containing no impurity atoms. The adequacy of this model due to the fact that almost all electronic devices are manufactured using nanotubes - is based on ideas about the structure and properties of ideal defectfree nanotubes [4]. The effective mass approximation and the Laplace transform for the density of states is employed [21].

This paper is organized as follows: in Sect. 2, the density of states and the grand thermodynamic potential of an electron gas on a tube are expressed in terms of the Jacobi elliptic theta function. In Sect. 3, the diamagnetic moment of an electron gas on the tube surface is obtained. 


\section{Density of states \\ and thermodynamic potential in terms of Jacobi elliptic $\Theta_{3}$ function}

The conduction electron energy spectrum in semiconductor nanotubes has a band nature. A small electron density near the band edge permits to use the effective mass approximation. Electron energy on a tube of radius $a$ in a magnetic field is $[13]$ :

$$
\varepsilon_{m k \sigma}=\varepsilon_{0}\left(m+\frac{\Phi}{\Phi_{0}}\right)^{2}+\frac{k^{2}}{2 m_{*}}+\sigma \mu_{\mathrm{B}} B,
$$

where $m_{*}$ is the effective mass of the electron, $\mu_{\mathrm{B}}=e /\left(2 m_{*} c\right)$ is the Bohr magneton, $m$ and $k$ are the projection of the angular momentum and electron momentum on the tube axis, $\sigma= \pm 1$ is the spin quantum number, $\varepsilon_{0}=1 /\left(2 m_{*} a^{2}\right)$ is the rotational quantum, $\Phi=\pi a^{2} B$ is the flux of magnetic induction $B$ in the tube (vector $\boldsymbol{B}$ directed along the axis of the tube) and $\Phi_{0}=2 \pi c / e$ is the flux quantum [5]. The quantum constant is hereinafter taken as equal to unity.

The grand thermodynamic potential of an electron gas is [26]:

$$
\Omega(S, T, \mu, B)=-\frac{1}{\beta} \int_{\varepsilon_{\min }}^{\infty} \mathrm{d} \varepsilon \nu(\varepsilon) \ln \left(1+\mathrm{e}^{\beta(\mu-\varepsilon)}\right),
$$

where $\beta=1 /\left(k_{\mathrm{B}} T\right)$ is the inverse temperature, $k_{\mathrm{B}}$ is the Boltzmann constant, $\mu$ is the chemical potential of the gas (where $\varepsilon_{\min }=-\mu_{\mathrm{B}} B$ ), $S=2 \pi a L$ is the surface area of the tube length $L$ :

$$
\nu(\varepsilon)=\sum_{m k \sigma} \delta\left(\varepsilon-\varepsilon_{m k \sigma}\right)
$$

is the density of states.

Using the Laplace transform, we represent the density of states in the form [13, 21, 27-30]:

$$
\nu(\varepsilon)=\frac{1}{2 \pi \mathrm{i}} \int_{b-\mathrm{i} \infty}^{b+\mathrm{i} \infty} \mathrm{d} \beta z(\beta) \mathrm{e}^{\beta \varepsilon},
$$

where

$$
z(\beta)=\sum_{m k \sigma} \mathrm{e}^{-\beta \varepsilon_{m k \sigma}}
$$

is the single-particle partition function, $\beta$ is the complex variable and $b>0$. By substituting (1) into (5), we obtain

$$
\begin{aligned}
& z(\beta)=\frac{L}{\pi} \sqrt{\frac{2 \pi m_{*}}{\beta}} \operatorname{ch}\left(\beta \mu_{\mathrm{B}} B\right) \\
& \times \sum_{m=-\infty}^{\infty} \exp \left(-\beta \varepsilon_{0}\left(m+\frac{\Phi}{\Phi_{0}}\right)^{2}\right) .
\end{aligned}
$$

The sum included above is [21, 31]:

$$
\begin{aligned}
& \sum_{m=-\infty}^{\infty} \exp \left(-\beta \varepsilon_{0}\left(m+\frac{\Phi}{\Phi_{0}}\right)^{2}\right)=\sqrt{\frac{\pi}{\beta \varepsilon_{0}}} \\
& \times\left(1+2 \sum_{l=1}^{\infty} \exp \left(-\frac{\pi^{2} l^{2}}{\beta \varepsilon_{0}}\right) \cos \left(2 \pi l \frac{\Phi}{\Phi_{0}}\right)\right) .
\end{aligned}
$$

The factor $\cos \left(2 \pi l \Phi / \Phi_{0}\right) \quad$ describes the Aharonov-Bohm oscillations of thermodynamic quantities considered in [13]. The factor included in brackets on the right-hand side of (7) is related to the Jacobi elliptic theta function $\Theta_{3}$ by the relation [31]:

$$
\Theta_{3}(v, x)=1+2 \sum_{l=1}^{\infty} \exp \left(-\pi^{2} l^{2} x\right) \cos (2 \pi l v),
$$

where $v=\Phi / \Phi_{0}$ and $x=1 /\left(\beta \varepsilon_{0}\right)$. As a result, the density of states (4) can be related as well to the function $\Theta_{3}$, i.e.,

$\nu(\varepsilon)=\frac{\nu_{0}}{2 \pi \mathrm{i}} \int_{b-\mathrm{i} \infty}^{b+\mathrm{i} \infty} \frac{\mathrm{d} \beta}{\beta} \operatorname{ch}\left(\beta \mu_{\mathrm{B}} B\right) \Theta_{3}\left(\frac{\Phi}{\Phi_{0}}, \frac{1}{\beta \varepsilon_{0}}\right) \mathrm{e}^{\beta \varepsilon}$.

Here, $\nu_{0}=m_{*} S / \pi$ means the density of states of a two-dimensional electron gas in the plane.

The grand thermodynamic potential in terms of an elliptic theta function is

$$
\begin{aligned}
\Omega & =-\frac{\nu_{0}}{2 \pi \mathrm{i}} \int_{\varepsilon_{\min }}^{\infty} \mathrm{d} \varepsilon \int_{b-\mathrm{i} \infty}^{b+\mathrm{i} \infty} \frac{\mathrm{d} \beta}{\beta^{2}} \operatorname{ch}\left(\beta \mu_{\mathrm{B}} B\right) \\
& \times \Theta_{3}\left(\frac{\Phi}{\Phi_{0}}, \frac{1}{\beta \varepsilon_{0}}\right) \ln \left(1+\mathrm{e}^{\beta(\mu-\varepsilon)}\right) \mathrm{e}^{\beta \varepsilon} .
\end{aligned}
$$

After double integration over parts of the integral over $\varepsilon,(10)$ can be presented in the form

$$
\Omega(S, T, \mu, B)=\int_{\varepsilon_{\min }}^{\infty} \mathrm{d} \varepsilon\left(-\frac{\mathrm{d} f(\varepsilon)}{\mathrm{d} \varepsilon}\right) \Omega(\varepsilon),
$$

where

$$
\Omega(\varepsilon)=-\frac{1}{2 \pi \mathrm{i}} \int_{b-\mathrm{i} \infty}^{b+\mathrm{i} \infty} \frac{\mathrm{d} \beta}{\beta^{2}} z(\beta) \mathrm{e}^{\beta \varepsilon},
$$

where $f(\varepsilon)$ is the Fermi-Dirac function.

\section{Landau diamagnetism}

In a general case, it is impossible to separate in (10) the monotonic and field-oscillating with $B$ terms in the thermodynamic functions of an electron gas. As in a three-dimensional case [26], it is possible in a weak magnetic field, when $\mu_{\mathrm{B}} B \ll k_{\mathrm{B}} T$ and $\Phi \ll \Phi_{0}$. From (8) and (9) it follows that the induction of the magnetic field $B$ is included in the hyperbolic cosine and factor $\cos \left(2 \pi l \Phi / \Phi_{0}\right)$ only. The factor $\operatorname{ch}\left(\beta \mu_{\mathrm{B}} B\right)$ is associated with the Pauli paramagnetism while the factor $\cos \left(2 \pi l \Phi / \Phi_{0}\right)$ is associated with the Aharonov-Bohm oscillations and the Landau diamagnetism. The AharonovBohm oscillations on a tube were studied in [13]. In the present paper, we focus on the Landau diamagnetism only.

Under the conditions $\mu_{\mathrm{B}} B \ll k_{\mathrm{B}} T$ and $\Phi \ll \Phi_{0}$, we select in the expansion $\operatorname{ch}\left(\beta \mu_{\mathrm{B}} B\right)$ and $\cos \left(2 \pi l \Phi / \Phi_{0}\right)$ the terms quadratic over the field. As a result, we obtain the paramagnetic $z_{p}(\beta)$ and diamagnetic $z_{d}(\beta)$ partition functions 


$$
\begin{aligned}
& z_{p}(\beta)=\frac{\nu_{0}}{2} \beta\left(\mu_{\mathrm{B}} B\right)^{2}, \\
& z_{d}(\beta)=-\frac{\nu_{0} \pi^{2}}{\beta \varepsilon_{0}^{2}}\left(\mu_{\mathrm{B}} B\right)^{2} \sum_{l=1}^{\infty} l^{2} \exp \left(-\frac{\pi^{2} l^{2}}{\beta \varepsilon_{0}}\right)
\end{aligned}
$$

The coefficient entering here is expressed through the Bohr magneton. The series in (14) is equal to

$\sum_{l=1}^{\infty} l^{2} \exp \left(-\frac{\pi^{2} l^{2}}{\beta \varepsilon_{0}}\right)=-\frac{\partial}{\partial\left(\frac{\pi^{2}}{\beta \varepsilon_{0}}\right)} \sum_{l=1}^{\infty} \exp \left(-\frac{\pi^{2} l^{2}}{\beta \varepsilon_{0}}\right)$.

The asymptotics of the series on the right-hand side of (15) and the functions $\Theta_{3}$ are known [32]:

$$
\sum_{l=-\infty}^{\infty} \mathrm{e}^{-l^{2} x}= \begin{cases}\sqrt{\frac{\pi}{x}}\left[1+2 \exp \left(-\frac{\pi^{2}}{x}\right)\right], & x \ll 1 \\ 1+2 \exp (-x), & x \gg 1\end{cases}
$$

The small-value $x$ corresponds to the lowtemperature region $\beta \varepsilon_{0} \gg 1$. In this area, we have

$$
\sum_{l=1}^{\infty} l^{2} \exp \left(-\frac{\pi^{2} l^{2}}{\beta \varepsilon_{0}}\right)=\frac{1}{4 \pi^{\frac{5}{2}}}\left(\beta \varepsilon_{0}\right)^{\frac{3}{2}}
$$

By substituting this expression into (14), we obtain

$$
z_{d}(\beta)=-\frac{\nu_{0}}{4} \sqrt{\frac{\beta}{\pi \varepsilon_{0}}}\left(\mu_{\mathrm{B}} B\right)^{2} .
$$

The substitution of this expression into (12) gives

$$
\Omega_{d}(\varepsilon)=\frac{\nu_{0}}{4} \frac{\left(\mu_{\mathrm{B}} B\right)^{2}}{\sqrt{\pi \varepsilon_{0}}} \frac{1}{2 \pi \mathrm{i}} \int_{b-\mathrm{i} \infty}^{b+\mathrm{i} \infty} \frac{\mathrm{d} \beta}{\beta^{2}} \mathrm{e}^{\beta \varepsilon} .
$$

The integral entering here is calculated by a loop $C_{0}$ in the complex plane of the variable $\beta$, going around the branch point $\beta=0$ (Fig. 1) [33]. It is calculated using the well-known formula

$$
\frac{1}{\Gamma(z)}=\frac{1}{2 \pi \mathrm{i}} \int_{C_{0}} \mathrm{~d} u \frac{\mathrm{e}^{u}}{u^{z}}
$$

As a result, we obtain

$$
\Omega_{d}(\varepsilon)=\frac{\nu_{0}}{2 \pi} \sqrt{\frac{\varepsilon}{\varepsilon_{0}}}\left(\mu_{\mathrm{B}} B\right)^{2},
$$

where the equality $\Gamma\left(\frac{3}{2}\right)=\frac{1}{2} \sqrt{\pi}$ is taken into account. Substituting (21) into (11), we obtain the diamagnetic contribution to the grand thermodynamic potential at low temperatures

$$
\Omega_{d}=\frac{\nu_{0}}{2 \pi} \sqrt{\frac{\mu}{\varepsilon_{0}}}\left(\mu_{\mathrm{B}} B\right)^{2} .
$$

The diamagnetic moment is

$$
M_{d}=-\frac{\partial \Omega_{d}}{\partial B}=-\frac{\nu_{0}}{\pi} \sqrt{\frac{\mu}{\varepsilon_{0}}} \mu_{\mathrm{B}}^{2} B .
$$

The meaning of this expression is that, with the appearance of the Aharonov-Bohm flow, circular persistent currents arise in the tube on its surface $[3,4,34]$. They create a magnetic field directed, according to Lenz's rule, in the direction opposite to the Aharonov-Bohm field. The ratio of this moment to the paramagnetic moment [13] is equal to $\frac{1}{\pi} \sqrt{\frac{\mu}{\varepsilon_{0}}}$. It contains both the material and geometric parameters of the nanotube.

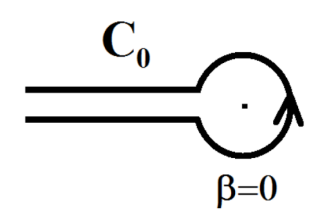

Fig. 1. The contour for calculating the integral (19).

\section{Discussion}

The equilibrium properties of the electron gas in nanotubes, as we have just seen, are mainly determined by the electron energy spectrum. This spectrum is, in turn, due to the geometry of the system.

In the scientific literature, for the theoretical study of the electron spectra of carbon nanotubes a model is used, which is obtained from the tightbinding approximation $[1,2]$, not taking into account the local curvature of the tube. The advantage of this model is that the crystal symmetry of the nanotube is adequately taken into account, while its disadvantage is associated with the neglect of the curvature of the tube. The energy band structure of individual nanotubes corresponds to the structure of a graphene sheet after the imposition of the Born-von Karman boundary conditions arising from its rolling into a cylinder. Thus, all armchair nanotubes are expected to exhibit metallic conduction, similar to the behavior of 2D graphene sheets $[1,2]$. The electronic properties of carbon nanotubes, in particular their magnetic properties, have been discussed theoretically based on a $\boldsymbol{k p}$ scheme in [8]. The magnetic moment is negative (diamagnetic) and its absolute value increases as a function of the magnetic field [8]. This result is in qualitative agreement with (23) for the diamagnetic moment obtained in this paper.

In this article, the Dirac energy spectrum of an electron on the surface of a nanotube is not used. We use the spectrum (1). This approximation allows to describe the properties of such systems qualitatively and often quantitatively. Thus, the results obtained in this paper can be used to describe the magnetic properties of armchair carbon nanotubes in the metal conduction regime.

\section{Conclusions}

The basic thermodynamic functions of an electron gas on the surface of a semiconductor nanotube in a magnetic field were calculated in [13]. It was shown, in particular, that thermodynamic quantities experience the Aharonov-Bohm oscillations with a change in the magnetic flux in the tube as well as oscillations existing in the absence of a magnetic field. They are due to quantization of the energy of the circular motion of electrons on the tube and the passage of quantized levels through the Fermi boundary when the parameters of the tube change. The spin magnetic moment of electrons was calculated in [13] but the diamagnetic moment was not calculated there. 
In this paper, the density of states and the grand thermodynamic potential of an electron gas on a semiconductor tube are expressed in terms of the Jacobi elliptic theta function. In a weak magnetic field at low temperatures, the diamagnetic moment of the electron gas is calculated. These results may be useful in studying the magnetic properties of a heterojunction with a two-dimensional electron gas bent into a cylinder.

The results obtained in this article are of considerable scientific interest. They can be used as the basis for the effective application of nanotubes in various fields of science and technology, e.g., for high-density magnetic recording as well as for creating polymer and composite materials with a given magnetic response.

\section{Acknowledgments}

The author expresses his gratitude to Professor A.M. Ermolaev for fruitful discussion of the results of this paper.

The author would also like to acknowledge the help of Dr. T.I. Rashba in preparing this manuscript.

\section{References}

[1] M.S. Dresselhaus, G. Dresselhaus, P.C. Eklund, Science of Fullerenes and Carbon Nanotubes, Academic, New York 1996.

[2] R. Saito, G. Dresselhaus, M.S. Dresselhaus, Physical Properties of Carbon Nanotubes, Imperial College Press, London 1998.

[3] Y. Imry, Introduction to Mesoscopic Physics, Oxford University Press, Oxford 2002.

[4] P.N. Dyachkov, Electronic Properties and Application of Nanotubes, Binom. Laboratory of Knowledge, Moscow 2012.

[5] I.O. Kulik, Sov. Phys. JETP 31, 1172 (1970).

[6] S. Iijima, Nature 354, 56 (1991).

[7] Yu.E. Lozovik, A.M. Popov, Physics Usp. 50, 749 (2007).

[8] H. Ajiki, T. Ando, Solid State Commun. 102, 135 (1997).

[9] V.A. Geyler, V.A. Iargulis, A.B. Shorohov, J. Exp. Theor. Phys. 88, 800 (1999).

[10] A.A. Zvyagin, I.V. Krive, Low Temp. Phys. 21, 533 (1995).

[11] A.I. Vedernikov, A.O. Govorov, A.V. Chaplik, J. Exp. Theor. Phys. 93, 853 (2001).

[12] P.A. Eminov, Yu.I. Sezonov, A.V. Al'pern, N.V. Sal'nikov, J. Exp. Theor. Phys. 103, 632 (2006).

[13] A.M. Ermolaev, G.I. Rashba, M.A. Solyanik, Eur. Phys. J. B73, 383 (2010).
[14] A.F. Slachmuylders, B. Partoens, W. Magnus, F.M. Peeters, J. Phys. Condens. Matter 18, 3951 (2006).

[15] A.M. Ermolaev, G.I. Rashba, Solid State Commun. 192, 79 (2014).

[16] A.M. Ermolaev, G.I. Rashba, M.A. Solyanik, Physica B 406, 2077 (2011).

[17] A.M. Ermolaev, G.I. Rashba, M.A. Solyanik, Phys. Solid State 53, 1594 (2011).

[18] A.M. Ermolaev, G.I. Rashba, M.A. Solyanik, Low Temp. Phys. 38, 957 (2012).

[19] A.M. Ermolaev, G.I. Rashba, Phys. Solid State 56, 1696 (2014).

[20] A.M. Ermolaev, G.I. Rashba, in: Handbook of Functional Nanomaterials, Vol. 4, Ed. M. Aliofkhazraei, Nova Sci. Publ., New York 2013, p. 213.

[21] A.M. Ermolaev, G.I. Rashba, in: Electron Gas: An Overview, Ed. T. Antonia, Nova Sci. Publ., New York 2019, Ch. 1, p. 1.

[22] G.I. Rashba, Acta Phys. Pol. A 136, 174 (2019).

[23] L. Wendler, V.M. Fomin, Z. Phys. B 96, 373 (1995).

[24] J. González, Phys. Rev. B 72, 073403 (2005).

[25] C. Chang, C. Ortix, Int. J. Mod. Phys. B 30, 1630016 (2016).

[26] L.D. Landau, A.I. Lifshitz, Course of Theoretical Physics, Vol. 5, Statistical Physics, 3rd ed., Butterworth-Heinemann, Oxford 1975.

[27] J.B. Rumer, J. Exp. Theor. Phys. 18, 1081 (1948).

[28] E.H. Sondheimer, A.H. Wilson, Proc. $R$. Soc. Lond. A210, 173 (1951).

[29] R. Kubo, Statistical Mechanics, NorthHolland, Amsterdam 1965.

[30] A.M. Ermolaev, G.I. Rashba, Introduction toward Statistical Physics and Thermodynamics, Kharkiv University Press, Kharkiv 2004.

[31] A.P. Prudnikov, Yu.A. Brichkov, O.I. Marichev, Integrals and Series, Gordon and Breach, New York 1986.

[32] M.A. Evgrafov, Asymptotic Estimates and Entire Functions, Dover, New York 2020.

[33] H. Bateman, A. Erdélyi, Higher Transcendental Functions, Vol. 1, McGraw-Hill, New York 1953.

[34] I.O. Kulik, Low Temp. Phys. 36, 841 (2010). 